\title{
Lattice QCD Calculations with $b$ Quarks: Status and Prospects
}

\section{Matthew Wingate*}

Department of Applied Mathematics and Theoretical Physics, University of Cambridge, Wilberforce Road, Cambridge CB3 OWA, UK

E-mail: M.Wingate@damtp.cam.ac.uk

This talk gives an overview of how lattice QCD calculations are influencing quark flavor physics. The first part of the talk focuses on the climb to higher precision; the second part surveys views along less-trodden paths.

The 13th International Conference on B-Physics at Hadron Machines (BEAUTY 2011) April 4-8, 2011 Amsterdam, The Netherlands

${ }^{*}$ Speaker. 


\section{Introduction}

It is a pleasure to give a brief review of lattice QCD (LQCD) calculations relevant for quark flavor physics, particularly $b$ physics. I am glad that the other theoretical review talks were able to review results from sum rules, models, and other nonperturbative approaches. Effective field theory plays a crucial role in LQCD, as in many other approaches; however, explicit discussion here will be limited. Even narrowing the scope of this talk to the lattice, I am thankful to the organizers for the constraints in speaking time and word count which preclude an encyclopedic review, thereby inviting an idiosyncratic one. In the following sections, I will say a few things about how LQCD results are being used in tests of CKM unitarity and $B_{s}$ mixing, and how I hope they can be used in rare $B_{(s)}$ and $\Lambda_{b}$ semileptonic decays.

Many attendees at this conference are familiar with remarks introducing LQCD. In the interest of brevity, allow me to just remind you that the following hierarchy of scales

$$
a \ll\left\{\frac{\hbar}{m_{b} c}, \frac{\hbar}{p_{\text {hadron }}}, \frac{\hbar c}{\Lambda_{\mathrm{QCD}}}, \frac{\hbar}{m_{\pi} c}\right\} \ll L
$$

is what is desired for the lattice spacing $a$ and box size $L$ in order to have uncertainties of a purely statistical nature (leaving aside the matching of regularization scheme-dependent quantities). However, what is achievable presently is closer to

$$
\frac{\hbar}{m_{b} c}<a \ll\left\{\frac{\hbar}{p_{\text {hadron }}}, \frac{\hbar c}{\Lambda_{\mathrm{QCD}}}\right\} \ll L<\frac{\hbar}{m_{\pi} c}
$$

with a restriction on $p_{\text {hadron }}$, the spatial momentum of any hadron in the lattice rest frame. We numerically solve the physics of the $\Lambda_{\mathrm{QCD}}$ scale; then we apply HQET or NRQCD to treat the physics of the $m_{b}$ scale, chiral perturbation theory to extrapolate to the $m_{\pi}$ scale and estimate finite $L$ effects, and Symanzik effective theory to reduce finite $a$ effects. Operator matching between lattice and continuum regularizations must often be done perturbatively, leading to another source of truncation error. The estimation and reduction of these uncertainties occupies most of the LQCD effort in calculating matrix elements.

\section{CKM unitarity tests}

I promised not to be encyclopedic, so I will not review individual lattice calculations here. Nevertheless, the list of recent unquenched calculations of form factors for $\left|V_{u b}\right|[1,2]$ and $\left|V_{c b}\right|$ [3, 4]; of the decay constants $f_{B_{(s)}}[5,6,7,8,9,10]$; and of the $\Delta B=2$ bag factors $B_{B_{(s)}}[11,6,8]$ demonstrates the vast LQCD effort being put into $B$ physics. Similar effort is being dedicated to $K$ physics (e.g. see [12]), in particular the $\Delta S=2$ bag factor $B_{K}[13,14,15,16,17,18,19]$, the $K \rightarrow \pi$ form factors $[20,21,22,23]$, and the decay constants $f_{\pi}$ and $f_{K}[24,25,26,27,28,29,30,31]$.

The primary goal of these calculations at present is to feed into CKM fits, checking for a single allowed region in the 4-dimensional space of $(\lambda, A, \bar{\rho}, \bar{\eta})$. As we will see later, however, the same quantities enter other observables, which are not precisely determined enough to constrain the CKM fits, but which might turn out to differ from their Standard Model predictions. Once the 
Standard Model has been supplanted, the hadronic matrix elements will still be needed in combination with whichever effective weak Hamiltonian replaces the one derived from the Standard Model.

The plots displaying global fits to the CKM parameters $\bar{\rho}$ and $\bar{\eta}$ have become iconic. Often we see them in the bold, outspoken colors used by CKMfitter. Other times they are displayed with the muted, conservative pastels used by the UTfit group. New groups are joining in the artistry, choosing their own color schemes. The talk by Lacker [32] discusses these fits in detail, but I want to briefly emphasize some differences in how three groups, CKMfitter [33], UTfit [34, 35], and Laiho, Lunghi, Van de Water (LLV) [36], use lattice results in their fits. (Sometimes a fit is done without LQCD data, which then allows a useful comparison between the fit results and lattice results.)

Before a quantity computed on the lattice can be taken as an input into the CKM fit, decisions must be made about which LQCD results to average together and how to propagate quoted uncertainties. Naturally, different groups tend to make different choices.

In the first respect, the difference between CKMfitter [33] and UTfit [34, 35] on one hand and LLV [36] on the other is whether to include lattice results which are missing a dynamical strange quark, i.e. $N_{f}=2$ calculations vs. the more physical $N_{f}=2+1$. Empirically, it has not yet been shown that the effects of the dynamical strange quark can be seen within present errors. However, one cannot argue the effects are perturbatively small, whereas one can for dynamical $c, b$, and $t$ quark effects. The LLV group take the latter view, not risking contamination from strange quark quenching effects. The disadvantage is that, by excluding some calculations, some other lattice systematics, e.g. choice of discretization, are not averaged over to the same extent. Therefore, I find the CKMfitter/UTfit approach of including $N_{f}=2$ results to be a reasonable compromise at the present time, although one will want to adopt the LLV approach ultimately. (In fact, for some quantities, UTfit actually use the LLV averages.)

The second difference between groups is how they treat the systematic errors assigned to individual lattice results. This is not surprising since, for example, some of these uncertainties arise due to the truncation of an expansion in a small parameter (e.g. the strong coupling $\alpha_{s}$ or $\left.\Lambda_{\mathrm{QCD}} / m_{B}\right)$. The size of the truncated terms is usually estimated to be equal to the next higher power of the small parameter times a number of $O(1)$. How to propagate that estimation in a statistical analysis is far from clear and groups make different choices. UTfit describe some lattice errors with a Gaussian distribution and others with a uniform distribution [37]. CKMfitter use their Rfit procedure which treats the statistical error as a Gaussian distribution and the systematic error as a flat distribution $[38,33]$. The total error is propagated forward as a broadened, flattened Gaussian. In contrast, LLV treat the quoted systematic error as an independent Gaussian distribution, to be combined in the usual way with the statistical error [36]. The effective difference between these approaches is that LLV usually quote more precise averages than CKMfitter. Whether one approach is too aggressive or the other is too conservative is a debate unlikely to reach a conclusion. In my experience, the dimensional analysis method of estimating truncation errors is reliable; one sees $3 \sigma$ discrepancies about as often as one would expect for a Gaussian distribution. Therefore, I think the LLV fits produce fair error estimates. Nevertheless it is understandable that one would want to be a bit more cautious with this type of theory error compared to a truly statistical error.

To give an idea how the different choices made by these groups can affect the inputs to CKM 
fits, let us look at the $K^{0}$ mixing parameter $\hat{B}_{K}$; respectively the groups quote $0.721(5)_{\text {stat }}(40)_{\text {sys }}$ (CKMfitter [33]), 0.725(27) (LLV [36]), and 0.731(7) stat (35) sys (UTfit [39]). The difference between a $5.5 \%$ uncertainty (or larger) and a $3.7 \%$ uncertainty in $\hat{B}_{K}$ actually has interesting consequences. With the smaller quoted uncertainty, a tantalizing discrepancy appears in CKM fits to $\varepsilon_{K}, \Delta M_{s} / \Delta M_{d}$, and the angle $\beta$ determined from $B \rightarrow(J / \psi) K_{S}[40,41,42,36]$. Also, with a $4 \%$ determination of $\hat{B}_{K}$, the uncertainty in $\left|V_{c b}\right|$ - in particular the exclusive/inclusive discrepancy becomes important. This tension may be due in part to NNLO corrections to $\varepsilon_{K}$ which have now been calculated [43].

While much LQCD activity is going forward, verifying presently quoted uncertainties, it is generally the case that new techniques are necessary to have a discrete improvement in precision. One area where we can expect improvement soon is in $B \rightarrow D^{(*)}$ form factors. Improved discretizations appropriate for charm quarks $[44,45]$ are now being implemented. As an indication of what can be achieved, the uncertainties in $f_{D_{(s)}}$ have been decreased to the $1-2 \%$ level $[25,46]$. We can expect that this development, along with the growing libraries of configurations being generated by the MILC Collaboration [28] and others, will produce an improved determination of $\mathscr{F} B \rightarrow D^{(*)}$. (Keep in mind that the uncertainties coming from the $b$ quark on the lattice will degrade the precision compared to $f_{D_{(s)}}$.) See talks by Lacker and Mannel [32, 47] which discuss CKM fits and their inputs in more detail.

\section{3. $B_{s}$ mixing}

One hot topic, already reviewed at this conference [48] has been the measurement by the D0 experiment of an anomalous like-sign di-muon asymmetry (due to one of the $B_{(s)}^{0} \bar{B}_{(s)}^{0}$ pair oscillating before decaying) which is 50 times larger than expected from the Standard Model [49, $50,51,52]$, a discrepancy at the $3 \sigma$ level:

$$
\begin{aligned}
& A_{S L}^{\mathrm{D} 0}=(-9.6 \pm 2.5 \pm 1.5) \times 10^{-3} \\
& A_{S L}^{\mathrm{LN}}=(-0.20 \pm 0.03) \times 10^{-3}
\end{aligned}
$$

where the asymmetry is roughly an average of flavor-specific asymmetries $A_{S L} \approx\left(a_{\mathrm{fs}}^{d}+a_{\mathrm{fs}}^{s}\right) / 2$. Given the size of the experimental signal and uncertainty, this is still something for the experimentalists to pin down. From my perspective though, it is interesting to note that the leading uncertainty in the theoretical estimate comes from the uncertainty in $\left|V_{u b} / V_{c b}\right|$ [51, 52]. In 2006/07 this uncertainty contributed to a $20 \%$ uncertainty in the flavor-specific asymmetry $a_{\mathrm{fs}}^{s}$. By $2010 / 11$ the $\left|V_{u b} / V_{c b}\right|$ error only contributed to a $12 \%$ uncertainty in $a_{\mathrm{fs}}^{s}$. The improvement in part came from progress combining much-improved theoretical and experimental work on $B \rightarrow \pi \ell v$ (see [47] here).

Turning to measurements which are limited by theoretical uncertainties, let me focus on Standard Model calculations of $\Delta M_{s}$ and $\Delta \Gamma_{s}$. The $B_{s}$ decay constant $f_{B_{s}}$ is the most important hadronic quantity entering the mass and width differences; it enters because one writes the matrix element of the 4-quark operators as the product of $f_{B_{s}}^{2}$ times known factors, which would be the result in the vacuum saturation approximation (VSA), and the "bag-factors" which represent deviations from the VSA. According to Lenz and Nierste's analysis, the contribution of the $f_{B_{s}}$ uncertainty to the 
errors in $\Delta M_{s}$ and $\Delta \Gamma_{s}$ has decreased from $33 \%$ to $13 \%$ in the past 5 years due to progress in lattice QCD [51, 52]. There is still much room for improvement; $f_{B_{s}}$ is still the most uncertain quantity in the calculation of $\Delta M_{s}$; the uncertainty in $B_{B_{s}}$ is even slightly less important than the $\left|V_{c b}\right|$ uncertainty, although the latter is more likely to be see a significant reduction soon. In the case of the width difference, $f_{B_{s}}$ is now precise enough that further reduction of uncertainty in $\Delta \Gamma_{s}$ requires a full lattice calculation of matrix elements through $O\left(\Lambda_{\mathrm{QCD}} / m_{b}\right)$. By itself, the numerical computation of these matrix elements would not be difficult; it is the perturbative matching calculation which requires significant, dedicated human effort.

\section{Rare decays}

We now turn from rare mixing to rare decays, already reviewed at this conference [53]. While the search for new physics in CKM fits is in some ways like a difficult, technical climb to the summit of Mt. Precision, the search for new physics in rare decays is like bushwhacking into the wilderness of overgrown backgrounds and nonfactorizable snakes. With such stealthy prey, we need hunters covering all paths.

The rare decays $B \rightarrow K^{*} \gamma$ and $B \rightarrow K^{(*)} \ell^{+} \ell^{-}$have been measured at the Tevatron and $B$ factories [54] and their branching fractions agree with Standard Model estimates [55, 56]. CDF has recently observed $B_{s} \rightarrow \phi \mu^{+} \mu^{-}$[57]. The LHC experiments (especially LHCb) expect to more precisely measure the lepton invariant-mass spectrum of these decays as well as other observables, some of which may reveal signs of physics beyond the Standard Model. While some of these observables are constructed so that hadronic quantities cancel, others will need precise determinations of hadronic matrix elements $[58,59]$.

Here lattice calculations can help by computing the form factors which parametrize the various hadronic matrix elements. Essentials of the calculations are the same as in the calculation of $\left\langle\pi\left(p^{\prime}\right)\left|V_{\mu}\right| B(p)\right\rangle$ [1], except a new matching calculation is necessary for the tensor operator [60].

The theory of rare $B_{(s)}$ decays at large recoil is under good control $[61,62,63]$. The main cause for concern has been nonlocal effects, primarily arising from the operator $Q_{2}=(\bar{s} b)_{V-A}(\bar{c} c)_{V-A}$, which creates a charmonium resonance before decaying to a lepton pair. At sufficiently large $q^{2}$ the matrix elements of non-local operators can be written in terms of the form factors in an operator product expansion. Rare, exclusive $b \rightarrow s \ell^{+} \ell^{-}$decays at low recoil look to be a promising new place to test the Standard Model.

This kinematic range is exactly where LQCD calculations can be done. As $q^{2}$ decreases, we first encounter growing discretization errors as the spatial momentum $\left|\mathbf{p}^{\prime}\right|$ of the final state meson becomes comparable to the inverse lattice spacing. While some tricks can be played [64], HQET errors also grow like $v \cdot p^{\prime} / m_{B}$ (where $v$ is the 4-velocity of the $B$ ). My collaborators and I have performed a calculation of the 10 form factors governing $B_{(s)}$ semileptonic and rare decays, including $S U(3)_{F}$ breaking effects. Preliminary results have appeared in conference proceedings, most recently CKM2010 [65]. Very soon we should be finalizing our calculations.

\section{Beautiful baryons}

The higher energy of LHC collisions will allow us to become better acquainted with the proper- 
ties of baryons which contain a $b$ quark, building upon the discoveries of the Tevatron experiments [54]. At the same the first experimental observations of many of these states were being made, the masses of the $b$-baryons were computed in unquenched lattice QCD [66, 67, 68, 69, 70, 71, 72]. A compilation of these results show good agreement among the computations, which use a variety of lattice formulations [73].

The study of $\Lambda_{b} \rightarrow \Lambda \ell^{+} \ell^{-}$at the LHC is also a promising one. The short distance physics should be the same as in $B \rightarrow K^{(*)} \ell^{+} \ell^{-}$. Nevertheless, with the $\Lambda$ in the final state, one hopes the $\Lambda$ baryon's polarization will increase sensitivity to any new right-handed couplings $[74,75]$. In LQCD one finds worse signal-to-noise ratios in correlation functions involving baryons compared to pseudoscalar mesons, so it would be difficult to extract all 12 form factors governing $\Lambda_{b} \rightarrow \Lambda$ decays. However, in the heavy quark limit these 12 reduce to only 2 [76]:

$$
\left\langle\Lambda\left(p^{\prime}\right)|\bar{s} \Gamma b| \Lambda_{b}(p)\right\rangle=\bar{u}_{\Lambda}\left(p^{\prime}\right)\left[F_{1}\left(q^{2}\right)+\downarrow F_{2}\left(q^{2}\right)\right] \Gamma u_{\Lambda_{b}}(p)
$$

where $q=p-p^{\prime}$. The time is ripe for LQCD study of matrix elements of this type.

\section{Conclusions}

We have every reason to believe there are natural explanations for the peculiarities of the Standard Model. It is clear we need as much information as possible - experimental data and theoretical calculations - in order to find overt signs of new physics in some places and to be sure of its absence in other places. We must pursue every path and leave no stone unturned.

\section{Acknowledgments}

I am grateful for support from the Science \& Technology Facilities Council and the Institute for Particle Physics Phenomenology, Durham University.

\section{References}

[1] E. Gulez et al., Phys. Rev. D73, 074502 (2006), hep-lat/0601021.

[2] J. A. Bailey et al., Phys. Rev. D79, 054507 (2009), 0811.3640.

[3] M. Okamoto et al., Nucl. Phys. Proc. Suppl. 140, 461 (2005), hep-lat/0409116.

[4] C. Bernard et al., Phys. Rev. D79, 014506 (2009), 0808.2519.

[5] M. Wingate et al., Phys. Rev. Lett. 92, 162001 (2004).

[6] E. Gamiz et al. (HPQCD Collaboration), Phys. Rev. D80, 014503 (2009), 0902.1815.

[7] B. Blossier et al. (ETM Collaboration), PoS LAT2009, 151 (2009), 0911.3757.

[8] C. Albertus et al., Phys. Rev. D82, 014505 (2010), 1001.2023.

[9] J. Simone et al. (Fermilab Lattice and MILC Collaborations), PoS LATTICE2010, 317 (2010).

[10] B. Blossier et al., PoS LATTICE2010, 308 (2010), 1012.1357.

[11] E. Dalgic et al., Phys. Rev. D76, 011501 (2007), hep-lat/0610104. 
[12] G. Colangelo et al. (FLAG working group) (2010), 1011.4408.

[13] E. Gamiz et al. (HPQCD), Phys. Rev. D73, 114502 (2006), hep-lat/0603023.

[14] S. Aoki et al. (JLQCD Collaboration), Phys. Rev. D77, 094503 (2008), 0801.4186.

[15] D. J. Antonio et al. (RBC), Phys. Rev. Lett. 100, 032001 (2008), hep-ph/0702042.

[16] C. Aubin, J. Laiho, and R. S. Van de Water, Phys. Rev. D81, 014507 (2010), 0905.3947.

[17] Y. Aoki et al. (2010), 1012.4178.

[18] T. Bae et al., Phys. Rev. D82, 114509 (2010), 1008.5179.

[19] M. Constantinou et al. (ETM Collaboration), Phys. Rev. D83, 014505 (2011), 1009.5606.

[20] P. Boyle et al., Phys. Rev. Lett. 100, 141601 (2008), 0710.5136.

[21] D. Brommel et al. (QCDSF collaboration), PoS LAT2007, 364 (2007), 0710.2100.

[22] V. Lubicz, F. Mescia, S. Simula, and C. Tarantino, Phys. Rev. D80, 111502 (2009), 0906.4728.

[23] P. Boyle et al., Eur. Phys. J. C69, 159 (2010), 1004.0886.

[24] S. Beane, P. Bedaque, K. Orginos, and M. Savage, Phys. Rev. D75, 094501 (2007), hep-lat/0606023.

[25] E. Follana, C. Davies, G. Lepage, and J. Shigemitsu, Phys. Rev. Lett. 100, 062002 (2008), 0706.1726.

[26] S. Aoki et al. (PACS-CS Collaboration), Phys. Rev. D79, 034503 (2009), 0807.1661.

[27] C. Aubin, J. Laiho, and R. S. Van de Water, PoS LATTICE2008, 105 (2008), 0810.4328.

[28] A. Bazavov et al., Rev. Mod. Phys. 82, 1349 (2010), 0903.3598.

[29] B. Blossier et al. (ETM Collaboration), JHEP 0907, 043 (2009), 0904.0954.

[30] J. Noaki et al. (JLQCD and TWQCD Collaborations), PoS LATTICE2010, 117 (2010).

[31] S. Durr, Z. Fodor, C. Hoelbling, S. Katz, S. Krieg, et al., Phys. Rev. D81, 054507 (2010), 1001.4692.

[32] H. Lacker, PoS BEAUTY2011, 032 (2011).

[33] V. Tisserand, in 44th Rencontres De Moriond EW (2009), 0905.1572.

[34] V. Lubicz and C. Tarantino, Nuovo Cim. B123, 674 (2008), 0807.4605.

[35] A. Bevan et al., Nucl. Phys. Proc. Suppl. 209, 109 (2010).

[36] J. Laiho, E. Lunghi, and R. S. Van de Water, Phys. Rev. D81, 034503 (2010), 0910.2928.

[37] M. Ciuchini, G. D’Agostini, E. Franco, V. Lubicz, G. Martinelli, et al., JHEP 0107, 013 (2001), hep-ph/0012308.

[38] A. Höcker, H. Lacker, S. Laplace, and F. Le Diberder, Eur. Phys. J. C21, 225 (2001), hep-ph/0104062.

[39] V. Lubicz, PoS LAT2009, 013 (2009), 1004.3473.

[40] A. J. Buras and D. Guadagnoli, Phys. Rev. D78, 033005 (2008), 0805.3887.

[41] E. Lunghi and A. Soni, Phys. Lett. B666, 162 (2008), 0803.4340.

[42] A. J. Buras and D. Guadagnoli, Phys. Rev. D79, 053010 (2009), 0901.2056.

[43] J. Brod and M. Gorbahn, Phys.Rev. D82, 094026 (2010), 1007.0684.

[44] E. Follana et al. (HPQCD), Phys. Rev. D75, 054502 (2007), hep-lat/0610092. 
[45] M. B. Oktay and A. S. Kronfeld, Phys. Rev. D78, 014504 (2008), 0803.0523.

[46] C. T. H. Davies et al., Phys. Rev. D82, 114504 (2010), 1008.4018.

[47] T. Mannel, PoS BEAUTY2011, 038 (2011).

[48] I. Bertram, PoS BEAUTY2011, 004 (2011).

[49] V. M. Abazov et al. (D0 Collaboration), Phys. Rev. D82, 032001 (2010), 1005.2757.

[50] V. M. Abazov et al. (D0 Collaboration), Phys. Rev. Lett. 105, 081801 (2010), 1007.0395.

[51] A. Lenz and U. Nierste, JHEP 06, 072 (2007), hep-ph/0612167.

[52] A. Lenz and U. Nierste (2011), 1102.4274.

[53] D. Straub, PoS BEAUTY2011, 049 (2011).

[54] D. Asner et al. (Heavy Flavor Averaging Group) (2010), 1010.1589.

[55] P. Ball, G. W. Jones, and R. Zwicky, Phys. Rev. D75, 054004 (2007), hep-ph/0612081.

[56] A. Ali, P. Ball, L. Handoko, and G. Hiller, Phys. Rev. D61, 074024 (2000), hep-ph/9910221.

[57] T. Aaltonen et al. (CDF Collaboration), Phys. Rev. Lett. 106, 161801 (2011), 1101.1028.

[58] C. Bobeth, G. Hiller, and D. van Dyk, JHEP 1007, 098 (2010), 1006.5013.

[59] C. Bobeth, G. Hiller, and D. van Dyk (2011), 1105.0376.

[60] E. Müller, A. Hart, and R. Horgan, Phys. Rev. D83, 034501 (2011), 1011.1215.

[61] G. Buchalla and G. Isidori, Nucl. Phys. B525, 333 (1998), hep-ph/9801456.

[62] B. Grinstein and D. Pirjol, Phys. Rev. D70, 114005 (2004).

[63] M. Beylich, G. Buchalla, and T. Feldmann, Eur.Phys.J. C71, 1635 (2011), 1101.5118.

[64] R. R. Horgan et al., Phys. Rev. D80, 074505 (2009), arXiv:0906.0945.

[65] Z. Liu et al. (2011), 1101.2726.

[66] H. Na and S. A. Gottlieb, PoS LAT2007, 124 (2007), 0710.1422.

[67] R. Lewis and R. Woloshyn, Phys. Rev. D79, 014502 (2009), 0806.4783.

[68] T. Burch et al., Phys. Rev. D79, 014504 (2009), 0809.1103.

[69] H. Na and S. Gottlieb, PoS LATTICE2008, 119 (2008), 0812.1235.

[70] W. Detmold, C.-J. Lin, and M. Wingate, Nucl.Phys. B818, 17 (2009), 0812.2583.

[71] H.-W. Lin, S. D. Cohen, N. Mathur, and K. Orginos, Phys. Rev. D80, 054027 (2009), 0905.4120.

[72] M. Wagner and C. Wiese (the ETM Collaboration) (2011), 1104.4921.

[73] R. Lewis, 12th International Conference on Meson-Nucleon Physics (2010), 1010.0889.

[74] C.-H. Chen and C. Geng, Phys. Rev. D63, 114024 (2001), hep-ph/0101171.

[75] T. Aliev, A. Ozpineci, and M. Savci, Nucl.Phys. B649, 168 (2003), hep-ph/0202120.

[76] T. Mannel, W. Roberts, and Z. Ryzak, Nucl.Phys. B355, 38 (1991). 